Reprod. Nutr. Dévelop. 1980, 20 (1 A), 139-154.

\title{
L'organe pinéal du Brochet (Esox lucius, L.). IV. Sérotonine endogène et activité monoamine oxydasique ; étude histochimique, ultracytochimique et pharmacologique
}

\author{
par J. FALCON *, MarieThérèse JUILLARD, J. P. COLLIN \\ Laboratoire de Zoologie ef Biologie Cellulaire, \\ ef Laboratoire associé ou CNRS no 290 \\ 40, avenue du Recteur-Pineau, 86022 Poitiers Cedex, France.
}

Summary. The pineal organ of the pike (Esox lucius L.). IV. Endogenous serotonin and monoamine oxidase activity : An histochemical, ultracytochemical and pharmacological study

The pineal organ of the pike was studied in natural conditions in autumn and winter (short days). Using fluorescence histochemistry and drug treatments, the agranular pool of 5-HTP/5-HT (5-hydroxytryptophan and/or 5-hydroxytryptamine), the melatonin precursor, was found in different receptor line cells (Falcon, 1979b). This result confirmed the possible existence of photoneuroendocrine cells. The 5-HTP/5-HT pool was also present, but less abundant, in ependymal (supportive = interstitial) cells. 5-HTP/5-HT fluorophores varied quantitatively, depending on the pineal region and the light-dark cycle. The distal part was poor in indoles as compared to the middle and proximal regions. 5-HTP/5-HT appeared to be maximum during the light phase and minimum during the dark phase; it was absent after $\mathrm{p}-\mathrm{CPA}$ and reserpine treatments and very abundant only in the middle and proximal regions after nialamide injection.

Rare nerve fibers with green fluorescence (indicating a catecholamine neurotransmitter) were found in the meningeal tissue outside the pineal organ ; they probably corresponded to those containing clear and dense-cored vesicles which were observed previously with electron microscopy (Falcon, 1979a).

Monoamine oxidase (MAO) activity was studied using light and electron microscopy. The oxidative deamination sites in the pineal epithelium were revealed in receptor line cells and interstitial cells, i.e. in the outer membrane of mitochondria, the membranes of the rough and smooth endoplasmic reticulum, the membranes of the nuclear envelope and the plasma membrane. The reaction product, indicative of MAO activity, was completely absent in nialamide-treated fish. Endogenous 5-HT may be the main substrate for MAO in the pike pineal organ.

\section{Introduction.}

D'après les résultats cytologiques (Falcon, 1979a, b; Falcon et Mocquard, 1979) et électrophysiologiques (Falcon et coll., en préparation), une fonction photosensorielle peut être actuellement attribuée à l'organe pinéal (ou épiphyse) du Brochet. Les don-

* Max-Planck-Institut für Physiologische und Klinische Forschung, W.G. Kerckhoff-Institut, Parkstrasse 1, 6350 Bad-Nauheim, BRD. 
nées concernant une fonction neuroendocrinienne mettant en jeu un (ou des ?) composé(s) de nature indolique (tel que la mélatonine) demeurent, chez le Brochet ef les Poissons d'une façon générale, préliminaires (réf. in Falcon, 1979a). Chez les Vertébrés primitifs, parmi les nombreux problèmes encore posés, reste celui du rôle exact de chacun des types cellulaires dans le métabolisme indolique. En particulier, il est intéressant d'établir si les fonctions photoréceptrice ef neuroendocrinienne sont dévolues à un seul type cellulaire (hypothèse de l'existence de cellules photoneuroendocriniennes : Collin, 1969, 1971 ; Oksche, 1971 ; Oksche et Hartwig, 1974, 1979) ou si, au contraire, le message sensoriel s'efface au profit d'un message neurohormonal dans des " photorécepteurs » en cours de rudimentation sensorielle et de recyclage fonctionnel, voisinant avec des photorécepteurs typiques.

Chez les Amniotes (Collin, 1979), on peut actuellement considérer que : (1) les photorécepteurs rudimentaires et les pinealocyłes sont responsables, selon un processus enzymatique, du métabolisme indolique et qu'ils élaborent, selon un mécanisme ribosomal classique, une sécrétion protéique stockée dans des vésicules à cœur dense, observées en microscopie électronique ; (2) les cellules interstitielles de type épendymaire participent, au moins, au stockage de la sérotonine et à la désamination oxydative.

Chez les Anamniotes, la sécrétion protéique de structure chimique inconnue ef de fonction hypothétique n'a pu être clairement mise en évidence au cours de nos récentes recherches sur les cellules de type photorécepteur (CTP) de l'organe pinéal de Brochet (Falcon, 1979b). En effet, l'élaboration de vésicules à cœur dense est très peu développée, du moins dans nos conditions expérimentales d'observation.

L'organe pinéal du Brochet (Owman et Rüdeberg, 1970) et celui de la Lamproie de Planer (Meiniel, 1979) sont les seuls à montrer jusqu'ici chez les Anamniotes, sans faire appel à la pharmacologie, un « pool » de 5-hydroxytryptophanne et/ou de 5-hydroxytryptamine (5-HTP/5-HT). Le catabolisme de la 5-HT représente, en outre, une voie très importante du métabolisme indolique, au moins chez les Vertébrés supérieurs (voir discussion).

L'ensemble de ces données justifie que, dans le présent travail, nous confirmions et précisions les résultats sur le « pool » de 5-HTP/5-HT en tenant compte, chez le Brochet, des données récentes sur la «zonation » morphologique de l'épiphyse (Falcon, 1979a) complétées par l'étude de la différenciation et de la répartition des chaînes bineuronales ou canaux sensoriels (Falcon, 1979b, c ; Falcon et Mocquard, 1979). De plus, nous avons cherché à établir, pour la première fois chez un Anamniote, la localisation des sites cellulaires de la désamination oxydative par la monoamine oxydase (MAO). Les résultats, ici présentés, serviront de support à de futures recherches sur le métabolisme indolique de l'organe pinéal du Brochet, par des méthodes variées et complémentaires.

\section{Matériel et méthodes.}

1) Matériel biologique (cf. Falcon, 1979a). — Les Brochets (Esox lucius, L.) ont été sacrifiés de février à mars 1977 et de septembre à novembre 1977 (environ 10 à 12 h d'éclairage journalier). 
2) Traitements pharmacologiques. - Les drogues suivantes, permettant d'intervenir au niveau des différentes étapes du métabolisme indolique ont été administrées par voie intrapéritonéale (entre les nageoires pelviennes) :

Le p-CPA (parachlorophénylalanine; Labkemi $A B$, Suède). Inhibiteur de la synthèse de la sérotonine (5-HT), à l'étape de l'hydroxylation du tryptophane : 3 injections de 300 à $400 \mathrm{mg} / \mathrm{kg}$ chacune, effectuées 72,48 et $24 \mathrm{~h}$ avant le sacrifice.

Le $\mathrm{Ro}_{4}-4602$ (N-D-Seryl-N-2, 3, 4, trihydroxybenzylhydrazine; Hoffman-La Roche, France), inhibiteur de la synthèse de la 5 -HT à l'étape de la décarboxylation du 5-hydroxytryptophanne (5-HTP) : $200 \mathrm{mg} / \mathrm{kg}$, en une injection, $4 \mathrm{~h}$ avant le sacrifice. Le nialamide (Niamide; Pfizer-Clin, France), inhibiteur de la monoamine oxydase (MAO), il provoque une augmentation du taux de 5-HT en fermant ses voies cataboliques : administration en une fois de $200 \mathrm{mg} / \mathrm{kg}, 18 \mathrm{~h}$ avant le sacrifice.

La réserpine (Serpasil ; Ciba, France) déplète les monamines de leurs sites de stockage : quatre injections de $5 \mathrm{mg} / \mathrm{kg}$ chacune, 48, 36, 24 ef $12 \mathrm{~h}$ avant le sacrifice.

3) Défection des monoamines par la technique de Falck et al. (1962). - La mise en évidence des monoamines endogènes de l'organe pinéal du Brochet a été réalisée par la méthode de Falck et Hillarp (Falck et al., 1962).

25 animaux ont été utilisés, soit 19 témoins et 6 traités.

a) Témoins. - Les sacrifices sont effectués à différents moments du nycthémère, soit pendant la phase nocturne (le soir entre 22 et $23 \mathrm{~h}: 3$ brochets, et entre 4 et $5 \mathrm{~h}$, le matin : 4 brochets), soit pendant la phase diurne (le matin, entre 10 et $11 \mathrm{~h}: 7$ brochets, et en fin d'après-midi entre 16 et $17 \mathrm{~h}: 5$ brochets).

b) Traités par les drogues. - Un par le p-CPA, 2 à la réserpine, 3 au nialamide (sacrifices effectués entre 10 et $11 \mathrm{~h}$ ou entre 16 et $17 \mathrm{~h}$ ).

c) Après sacrifice des animaux, les encéphales sont prélevés in toto, immédiatement immergés (pendant $20 \mathrm{sec}$ ) dans l'isopentane juste décongelé, puis dans l'azote liquide (5 min minimum). Les pièces sont ensuite lyophilisées 48 à $72 \mathrm{~h}$, à $-40^{\circ} \mathrm{C}$, à l'aide d'un lyophilisateur Edwards Pearse EPD 3 (Grande-Bretagne), puis traitées (pour la condensation des amines) aux vapeurs de paraformaldéhyde (Merck), équilibrées à 70 p. 100 d'humidité, dans un bocal hermétiquement clos, placé pendant $3 \mathrm{~h}$, dans une étuve $\mathrm{a}+80^{\circ} \mathrm{C}$.

Les pièces sont incluses sous vide dans la cytoparaffine dégazée. Les coupes, de 5 à $8 \mu \mathrm{m}$ d'épaisseur, montées dans l'entellan (Merck), sont immédiatement observées au microscope Leitz-Wetzlar équipé d'une lampe à vapeurs de mercure HBO 200 et d'une combinaison de filtres (BG 12/TK 510/K 515).

Les préparations sont photographiées sur film Rayoscope RY-135-50 (32 Din, 800 Asa, Kodak).

4) Technique ultracytochimique. - Mettant à profit les propriétés réductrices des monoamines (catécholamines ef sérotonine ; Hakanson et al., 1971 ; Juillard et Collin, 1976 ; Collin et al., 1977) en présence d'un sel d'argent, nous avons appliqué la technique argentaffine.

Treize brochets sont utilisés ( 2 témoins, 5 traités au $\mathrm{P}-\mathrm{CPA}, 2$ au $\mathrm{Ro}_{4}-4602,2$ au nialamide, 2 à la réserpine), sacrifiés pendant la période diurne, entre 8 h et 13 h 30 . 
Les épiphyses sont fixées au glutaraldéhyde à 2,5 p. 100 dans le tampon phosphate $(0,1 \mathrm{M}, \mathrm{pH} 7,4)$ pendant $1 \mathrm{~h} 30$, puis rincées dans le même tampon, déshydratées et incluses dans l'Epon 812.

Les coupes ultrafines $(600$ à $900 \AA)$ sont recueillies sur grilles de nickel de 300 mesh et mises à flotter sur le liquide de Fontana (Juillard et Collin, 1976 ; Collin ef al.,1977).

L'incubation a lieu 1 à $4 \mathrm{~h}$ à $+60^{\circ} \mathrm{C}$, à l'obscurité et en atmosphère humide.

5) Mise en évidence d'une activité monoamine oxydasique (MAO).

a) Détection de l'activité MAO en microscopie photonique.

La technique de Glenner et al. (1957, réf. in Gabe, 1968), basée sur la réduction des sels de tétrazolium, est appliquée.

Les épiphyses de 5 brochets (dont un traité au nialamide) sont congelées à $-20^{\circ} \mathrm{C}$, immédiatement après la dissection, réalisée à $10 \mathrm{~h}$ ou à $16 \mathrm{~h}$. Elles sont débitées (cryostat Harris, Mass.) en coupes transversales de $16 \mu \mathrm{m}$ d'épaisseur, à $-20^{\circ} \mathrm{C}$ et recueillies sur lames de verre, préalablement gélatinées. Après 10 à 20 min de séchage à la température ambiante, les coupes sont recouvertes du milieu d'incubation suivant : chlorhydrate de tryplamine (Fluka, Suisse) : $25 \mathrm{mg}$; sulfate de sodium anhydre :

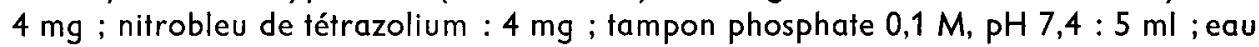
bidistillée : $15 \mathrm{ml}$.

L'incubation a lieu à l'obscurité, à $+37^{\circ} \mathrm{C}$, en atmosphère humide, pendant $1 \mathrm{~h}$. Après rinçage, les préparations sont fixées $1 \mathrm{~h}$ au glutaraldéhyde à $6 \mathrm{p}$. 100 dans le tampon phosphate $0,1 \mathrm{M}, \mathrm{pH} 7,4$, rincées à nouveau, déshydratées à l'acétone et montées dans le Permount.

b) Détection de l'activité MAO en microscopie électronique, (Shannon ef al., 1974 ; Müller et Da Lage, 1977).

La méthode est basée sur le même principe que précédemment, mais le sel utilisé, le BSPT (2-(2'benzothiazolyl)-5-styryl-3-(4'-phthalhydrazidyl) tétrazolium chloride ; Polysciences Inc., Penn.) est non osmiophile et donne à l'état réduit un précipité de formazan osmiophile. La tryptamine (chlorhydrate de) est choisie comme substrat.

Dans la recherche à la fois du maintien de l'intégrité des tissus et d'une bonne conservation de l'activité monoamine oxydasique, plusieurs prolocoles de fixation ont été utilisés :

- les épiphyses de 2 brochets (dont un « nialamidé ») sont fixées pendant $1 \mathrm{~h}$, à $+4^{\circ} \mathrm{C}$ dans le formaldéhyde dépolymérisé (solution à 1 p. 100 dans le tampon phosphate $0,1 \mathrm{M} ; \mathrm{pH} 7,4) ; 1$ autre épiphyse n'est fixée que pendant $5 \mathrm{~min}$.;

- deux épiphyses sont fixées respectivement $5 \mathrm{~min}$ et $1 \mathrm{~h}$, à $+4^{\circ} \mathrm{C}$, dans le glutaraldéhyde à 1 p. 100 (dans le tampon phosphate $0,1 \mathrm{M} ; \mathrm{pH} 7,4$ ).

Les épiphyses sont ensuite enrobées dans l'agar-agar ef débitées en blocs de $100 \mu \mathrm{m}$ à l'aide d'un sectionneur de tissus (Sorvall TC2, Newton, Conn.) ou bien sont coupées selon le plan sagittal, à l'aide d'une lame de rasoir. Enfin, elles sont rincées 18 à $24 \mathrm{~h}$ dans le tampon.

Deux milieux d'incubation sont préparés de la façon suivante :

1 er milieu : chlorhydrate de tryptamine, $25 \mathrm{mg}$; sulfate de sodium anhydre, $4 \mathrm{mg}$; sucrose, $4 \mathrm{mg}$; BSPT, $5 \mathrm{mg}$; diméthyl formamide, $0,25 \mathrm{ml}$; tampon phosphate $(0,1 \mathrm{M}$; $\mathrm{pH} 8), 10 \mathrm{ml}$; eau bidistillée, $10 \mathrm{ml}$. 
Juste avant l'emploi, le tampon et l'eau bidistillée sont versés dans un premier flacon contenant le chlorhydrate de tryptamine, le sulfate de sodium et le sucrose. Le BSPT est dissous dans le diméthyl formamide, dans un deuxième flacon. Les contenus de chaque flacon sont mélangés, puis filtrés.

2e milieu : préparé comme précédemment, à l'exception du chlorhydrate de tryptamine qui n'est pas présent dans le premier flacon.

Après rinçage, les sections sont mises à incuber $1 \mathrm{~h}$ à $+4^{\circ} \mathrm{C}$ dans le milieu sans substrat, puis $1 \mathrm{~h}$ dans le milieu avec substrat à $+20^{\circ} \mathrm{C}$ (des sections témoins, autres que celles de l'animal traité au nialamide, incubent dans un milieu sans substrat).

Toutes les sections sont ensuite rincées dans le tampon phosphate $(0,1 \mathrm{M} ; \mathrm{pH} 7,4)$ puis post-fixées dans le tétroxyde d'osmium pendant $2 \mathrm{~h}$, à $+40^{\circ} \mathrm{C}$. Enfin elles sont rincées, déshydratées par les alcools et incluses dans l'Epon 812 (en évitant le passage dans l'oxyde de propylène).

\section{Résultats.}

1) Détection des indolamines par la technique de Falck et al. - L'épithélium épiphysaire, traité selon la technique de Falck ef al., présente des fluorophores jaunes dont l'intensité varie selon les cellules considérées et l'heure du sacrifice. Ces fluorophores sont labiles (caractère de spécificité du 5-HTP et de la 5-HT).

Lorsque les animaux sont sacrifiés à 10 ou à $16 \mathrm{~h}$, s'observent dans la région moyenne des « îlots » fluorescents (type 1) et polymorphes (fig. 1) dont certains apparaissent formés de plusieurs unités constituant des « rosettes» (type 2 : fig. 2).

Alors que les « îlots » de type 1 se situent aux différents niveaux de l'épithélium, les « rosettes » occupent généralement une position basale.

Dans la région proximale, les « îlots » des 2 types sont moins abondants (fig. 1) ; ils n'ont pas été observés dans la région distale où de nombreuses cellules sont caractérisées par des fluorophores jaunes, diffus et peu intenses (fig. 3).

Sur des coupes favorables, longitudinales, il nous a été permis d'observer des fluorophores jaunes (fig. 4), plus ou moins intenses, dans le segment interne et les pédicules basaux de CTP. II ressort de nos observations que les « îlots 》 polymorphes de type 1 pourraient représenter des sections selon des plans variés, réalisées au niveau des différents compartiments des CTP, alors que les rosettes pourraient correspondre (de par leur taille et localisation dans l'épithélium) à des groupements de pédicules basaux (observés antérieurement en microscopie électronique, Falcon, 1979b). Si notre interprétation est correcte, il semblerait que les CTP soient plus intensément fluorescentes que les cellules interstitielles de type épendymaire (CIE). La technique radioautographique doit confirmer ou infirmer notre hypothèse.

Parmi les méninges qui circonscrivent l'organe pinéal et autour des capillaires, nous avons parfois identifié des fibres à fluorescence verte (fig. 5) ; mais elles ne sont jamais observées dans le parenchyme épiphysaire.

Le traitement au nialamide augmente intensément la fluorescence jaune, qui concerne CTP et CIE dans les régions moyenne et proximale (fig. 6,7$)$, mais ne semble pas modifier celle de la région distale (fig. 7).

Les traitements au P-CPA ou à la réserpine suppriment toute fluorescence dans l'épithélium épiphysaire (fig. 8, 9). 
Lorsque des animaux - non traités par des drogues - sont sacrifiés à $22 \mathrm{~h}$ ou à $4 \mathrm{~h}$, seuls quelques rares îlots fluorescents sont observés dans l'épithélium épiphysaire de la région moyenne (fig. 10).

2) Mise en évidence qualitative des indolamines par la méthode argentaffine. - Aucun résultał n'a été obtenu par cette méthode, malgré plusieurs tentatives.

3) Mise en évidence d'une activité MAO dans l'épithélium épiphysaire.

a) En microscopie photonique : après traitement, les coupes - réalisées dans les épiphyses des animaux témoins - présentent des précipités de formazan qui traduisent une activité monoamine oxydasique spécifique (absente chez les Poissons traités au nialamide : fig. 11). L'intensité de tels précipités varie selon les régions de l'épithélium et l'heure du sacrifice.

- Animaux sacrifiés à $10 \mathrm{~h}$ : l'activité MAO est localisée dans certaines zones de la région distale (fig. 12) et surtout moyenne (fig. 13). Aucune activité n'est décelée dans la région proximale (fig. 14).

\section{PLANCHE I}

Hisfofluorescence de l'organe pinéal du Brochet (méthode de Falck et al.)

FIG. 1. - Régions moyenne et proximale (Témoin sacrifié à $10 \mathrm{~h}$ ). Des « îlots» fluorescents (petites têtes de flèches) sont observés dans la région moyenne (mpi). On remarque également la fluorescence légèrement moins intense de certaines cellules. Dans la région proximale (ppi), les « îlots 》 sont plus rares et la fluorescence diffuse moins intense (flèches : mastocytes fluorescents; tête de flèche large : méninges autofluorescentes) $(\times 84)$.

FIG. 2. - «llots » intensément fluorescents constituant 2 rosettes (têtes de flèches). Noter la fluorescence faible et diffuse d'autres cellules (petite flèche) $(\times 300)$.

FIG. 3. - Régions moyenne et disfale (Témoin sacrifié à $10 \mathrm{~h}$ ). Contrairement à la région moyenne (mpi et têtes de flèches), aucun «îloł » fluorescent n'est observé dans la région distale (dpi) où les cellules montrent cependant une fluorescence générale de faible intensité (petites flèches) ( $\times$ 59).

FIG. 4. - Un « îlot» fluorescent est localisé à la base d'une CTP (tête de flèche). Dans le reste de la cellule s'observent des points ou des zones à fluorescence moins intense (petites flèches) dans le segment interne (is) $(\times 960)$.

FIG. 5. - Groupe de fibres à fluorescence verte dans la région périépiphysaire (flèches) $(\times 480)$.

FIGS. 6 ef 7. - Animal «nialamidé ». Toutes les cellules sont fluorescentes dans la région moyenne (mpi), contrairement à la région distale (dpi) où aucune modification n'est décelée par rapport aux animaux témoins (tête de flèche : méninges autofluorescentes) (Fig. $6: \times 80$; Fig. 7 : $\times 53)$.

FIG. 8. - Animal traité au p-CPA, et FIG 9 : Animal « réserpiné ». Aucune fluorescence n'est observée dans l'épithélium épiphysaire (tête de flèche : méninges autofluorescentes) (Fig. $8: \times 164$; Fig. $9: \times 45)$.

FIG. 10. - Animal témoin, sacrifié à $4 \mathrm{~h}$ du matin. Seuls quelques très rares îlots fluorescents sont observés (flèches) dans la région moyenne (tête de flèche : méninges autofluorescentes) $(\times 59)$. 


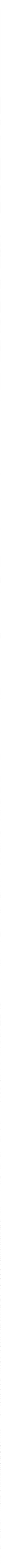


- Animaux sacrifiés à $16 \mathrm{~h}$ : l'épithélium épiphysaire dans sa totalité, de même que celui du sac dorsal, sont le siège d'une intense activité MAO (fig. 15, 16).

b) En microscopie électronique : au cours de l'après-midi, l'activité MAO — visualisée par des précipités denses aux électrons - est révélée de façon satisfaisante après fixation au glutaraldéhyde à 1 p. 100 pendant 5 min ou au formaldéhyde à 1 p. 100 pendant $1 \mathrm{~h}$; ce dernier fixateur n'assure pas une bonne préservation des fissus.

L'activité MAO est mise en évidence dans la région moyenne (seule éfudiée) de l'épiphyse ef plus précisément dans les CTP ef les CIE :

- Sur la membrane mitochondriale externe : les précipités, signalant l'activité MAO, ne concernent pas toutes les mitochondries des CTP (fig. 17) et des CIE. Dans ces dernières, les mitochondries, caractérisées par une activité MAO, sont plus nombreuses au pôle basal de la cellule (fig. 18) et la totalité de la membrane externe des mitochondries réactives n'est pas toujours marquée par l'activité enzymatique.

- Sur la membrane externe et parfois interne de l'enveloppe nucléaire des deux types cellulaires (fig. 17, 18).

- Sur les membranes du réticulum lisse el granulaire (fig. 17) ; les citernes ergastoplasmiques dans les CIE sont toutefois peu marquées (fig. 19).

- Sur la membrane plasmique des CIE (fig. 20) et surtout sur celle des CTP (fig.17, 20. 21) et en particulier dans la zone des pédicules (fig. 20).

Enfin, nous avons également observé des précipités sur la membrane mitochondriale externe et les membranes de l'enveloppe nucléaire de l'endothélium des capillaires.

Aucune réaction n'est enregistrée après incubation dans un milieu sans substrat ou dans l'épithélium épiphysaire de l'animal traité au nialamide.

\section{PLANCHE II}

Activité monoamine oxydasique de l'organe pinéal du Brochet (étude en microscopie photonique par la méthode de Glenner et al.).

FIG. 11. - Coupe témoin, animal traité au nialamide : aucun précipité de formazan n'est observé $(\times 120)$.

FIGS. 12 à 14. - Animaux sacrifiés à $10 \mathrm{~h}$.

Fig. 12 : région distale ; l'activité MAO semble localisée dans certaines parties de l'épithélium de la région distale (têtes de flèches) $(\times 197)$.

Fig. 13 : région moyenne : plus importante que dans la région distale, l'activité MAO ne concerne cependant que certaines zones de l'épithélium de cette région (têtes de flèches) $(\times 190)$.

Fig. 14 : tige épiphysaire (pis) ; aucune activité MAO n'est décelée $(\times 226)$ (ds : sac dorsal ; pl : lumière épiphysaire).

FIGS. 15 et 16. - Animaux socrifiés d̀ $16 \mathrm{~h}$.

Fig. 15 : région moyenne : l'activité MAO concerne tout l'épithélium de cette région ( $\times 328$ ).

Fig. 16 : région proximale : la tige épiphysaire (pis) ainsi que l'épithélium du sac dorsal (ds) sont le siège d'une activité MAO ( $p l$ : lumière épiphysaire) $(\times 328)$. 
今ै

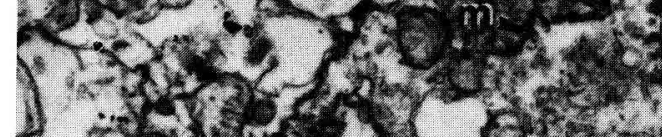

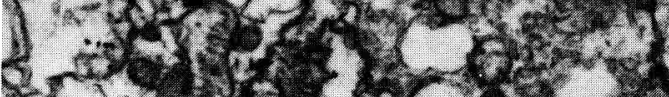

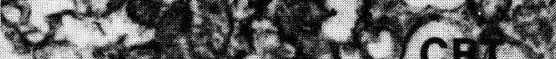

athos (ch)

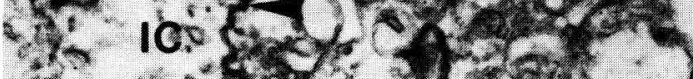

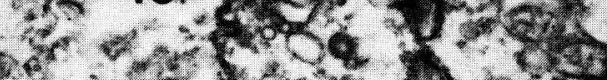

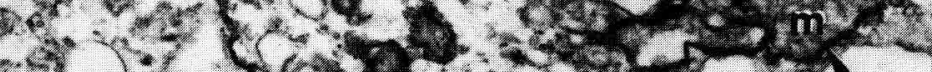

2a-r. ris.

6.2 2060

$1 \mathrm{C}$.

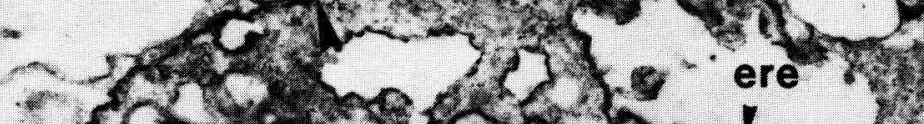

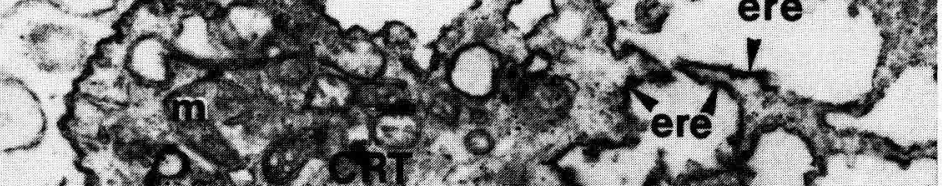

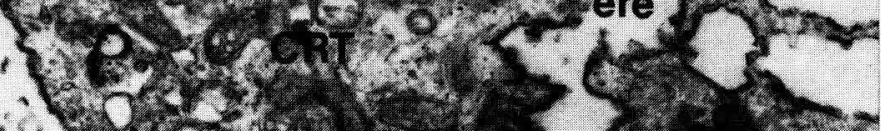

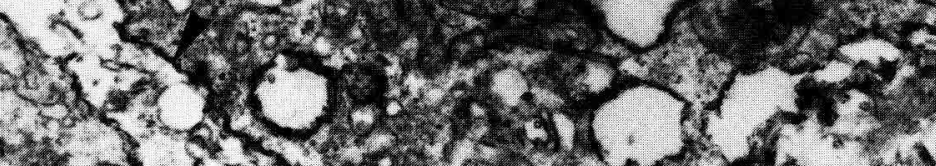

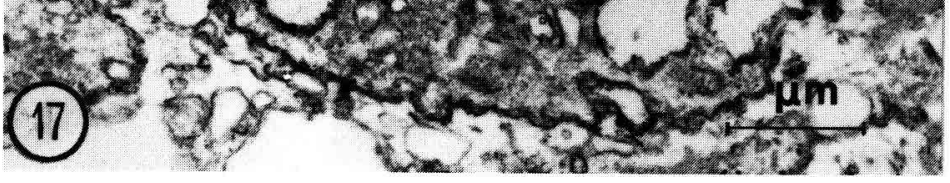

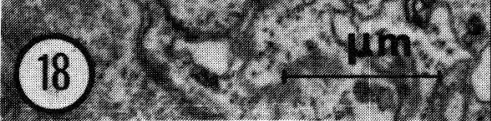

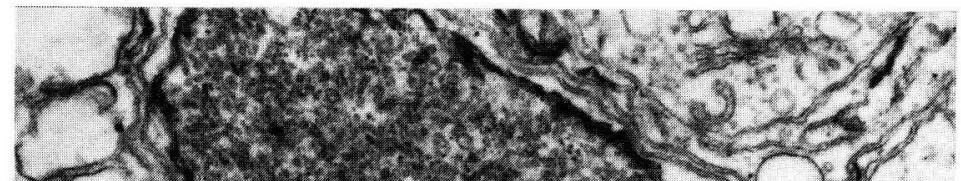
IC (20)

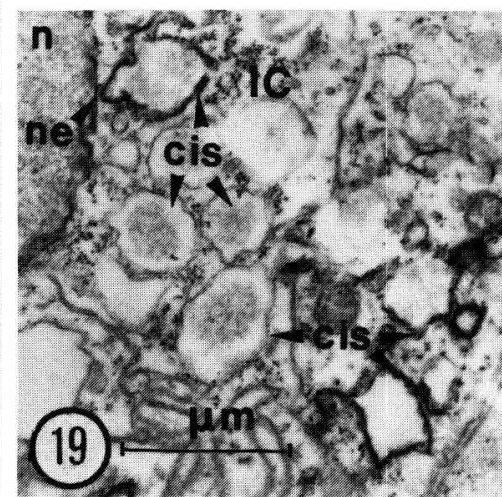
s. $10.5 \%$ <4 48000 ice (cos. ny \{ $5 \mathrm{~S}^{2} \mathrm{ic}$ [us $5-9$

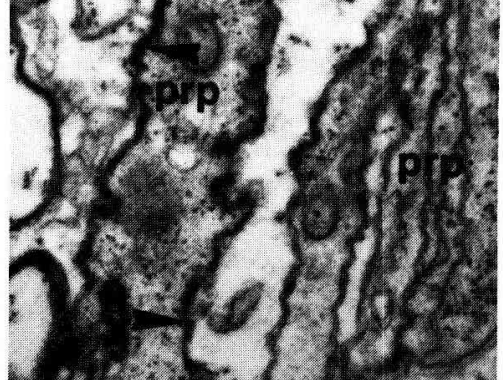

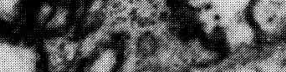

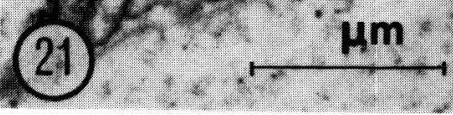




\section{Discussion.}

Les preuves d'une activité neurohormonale de l'organe pinéal des Téléostéens et la mise en évidence par des méthodes biochimiques de certaines enzymes du métabolisme indolique ef de la mélatonine (réf. in Falcon, 1979a) nous ont incités à rechercher, chez le Brochei, les sites cellulaires du métabolisme indolique. Dans des cellules, dont on peut soupçonner le caractère récepto-sécrétoire (Collin, 1979; Collin et Oksche, en préparation), la connaissance de la nature du (des) stimulus et de ses (leurs) modalités de contrôle du métabolisme indolique et de la libération des molécules actives (5-méthoxy-indoles : mélatonine et 5-méthoxytryptophol) est primordiale. Compte tenu des résultats acquis dans le présent travail, la discussion sera limitée ici à certains aspects qualitatifs et quantitatifs (1) du pool de 5-HTP/5-HT précurseur des 5-méthoxy-indoles (revue de Wurtman et al., 1968) et (2) des sites de la désamination oxydative.

1) Le « pool 》 de 5-HTP/5-HT : sites de stockage et variations dans les conditions naturelles et expérimentales. - Rappelons tout d'abord que la synthèse de la 5-HT est réalisée à partir du tryptophanne circulant qui est hydroxylé en 5-HTP par la tryptophanne-5mono-oxygénase de cellules pinéaliennes ; le 5-HTP est ensuite décarboxylé et transformé en 5-HT grâce à une L-amino-acide aromatique décarboxylase (AAAD) (rev. de Wurtman et al., 1968 ; Quay, 1974).

Dans les conditions automnales et hivernales, précisées antérieurement (cf. Matériel ef méthodes), l'organe pinéal du Brochel montre, par la technique de Falck et al. (1962), des fluorophores jaunes caractéristiques de la présence de 5-HTP/5-HT. L'étude microspectrofluorimétrique de ces fluorophores (Owman et Rüdeberg, 1970)

\section{PLANCHE III}

Activité monoamine oxydasique

(étude en microscopie électronique par la méthode de Shannon et al.).

FIG. 17. - Cellules de type photorécepteur (CRT) : l'activité MAO est localisée au niveau des membranes de l'enveloppe nucléaire (ne), de certaines membranes mitochondriales externes $(m)$, du réticulum endoplasmique (ere) et de la membrane cellulaire (grande tête de flèche) (IC : cellule interstitielle; $n$ : noyau) $(\times 13500)$.

FIG. 18. - Cellules interstifielles (IC) : la réaction est ici localisée au niveau de la membrane externe de l'enveloppe nucléaire ( $n$ e) et des membranes mitochondriales externes $(\mathrm{m})$ ( $b$ l : lamelle basale ; $n$ : noyau) $(\times 16000)$.

FIG. 19. - Cellule interstitielle (IC) : précipités sur l'enveloppe nucléaire (ne) et certaines membranes des citernes du réticulum endoplasmique granulaire (cis), ( $n$ : noyau) $(\times 17500)$.

FIG. 20. - Activité MAO sur la membrane des pédicules de photorécepteurs ( $p p$ ) et (à un degré moindre) sur celle de certaines cellules interstitielles $(I C)(\times 16800)$.

FIG. 21. - Activité MAO sur la membrane d'un pédicule de photorécepteur rudimentaire ( $p r p$ ) prenant appui sur la lamelle basale (IC : cellule interstitielle) $(\times 20000)$. 
de même que les variations de leur taux, induites après l'injection de P-CPA, réserpine et de nialamide renforcent la conclusion que les composés fluorescents visualisent un stockage de 5-HTP/5-HT sans qu'il soit pour autant possible de dissocier ces deux composés (revue de Björklund et al., 1975). De plus, contrairement aux Mammifères, chez lesquels la fluorescence jaune correspond essentiellement, sinon uniquement, à de la 5-HT, la décarboxylation, chez les Vertébrés inférieurs, pourrait être une étape limitante (Wilhoft et Quay, 1965) de telle sorte qu'en l'absence de recherches biochimiques, un « pool » de 5-HTP/5-HT (au lieu de 5-HT) paraît davantage justifié chez ces derniers.

Bien que dans nos conditions expérimentales les réactions enregistrées apparaissent sensiblement moins intenses que celles obtenves par Owman et Rüdeberg (1970), nos résultats viennent confirmer les données de ces auteurs. Cette différence d'intensité peut être liée à l'âge des animaux (ceux que nous avons utilisés étaient jeunes), mais plus vraisemblablement à la date du sacrifice (ici pendant les périodes automnale ef hivernale). En effet, des variations saisonnières et un rythme circannuel du taux de 5-HT ont pu être établis chez les Reptiles lacertiliens et chéloniens (Petit, 1976 ; VivienRoels, 1976 ; Vivien-Roels ef al., 1978).

Notre éfude apporte également des nuances ef des compléments à celle de nos prédécesseurs : dans nos conditions d'observation, seule la région moyenne de l'épiphyse présente des « îlots » fluorescents en quantité notable ; ils sont moins nombreux dans la région proximale, absents dans la région distale. Ceci permet d'envisager des variations régionales dans le stockage du 5-HTP/5-HT, réflétant par ailleurs certaines différences dans l'activité des cellules impliquées dans le métabolisme indolique. Ces différences apparaissent encore après injection de nialamide, drogue qui entraîne une augmentation de la concentration de la $5-\mathrm{HT}$ dans toutes les cellules des régions proximale et moyenne, mais n'apporte pas de changement dans la région distale. Comme les fluorophores jaunes sont détectés (en plus ou moins grande quantité) dans tout l'organe pinéal, il faut en déduire que toutes les régions de l'organe paraissent concernées par le métabolisme indolique. Cependant, les fluorophores jaunes, localisés dans les deux catégories cellulaires CTP et CIE, semblent présents en quantité plus importante dans les CTP (« îlots » fluorescents ef formation en « rosettes », voir résultats).

Les variations des fluorophores, au cours du nycthémère, indiquent l'existence d'un rythme du taux de 5-HTP/5-HT dans l'organe pinéal du Brochet avec un maximum pendant la période diurne et un minimum pendant la phase nocturne. Ceci est à rapprocher des observations faites chez les Sauropsidés (Quay, 1966 ; Petit, 1976 ; Vivien-Roels, 1976 ; Vivien-Roels et al., 1978 ; voir également références correspondantes) et des Mammifères (réf. in Quay, 1974) et laisse à penser que la mélatonine serait produite à partir de la 5-HT (réf. in Wurtman ef al., 1968 ; Falcon, $1979 a$ ) surtout pendant la phase obscure. Les moments du nycthémère où le taux de 5-HT est maximum ou minimum varient selon les espèces.

En bref, nos recherches, par la technique de fluorescence, ont contribué (1) à établir la présence et la localisation du 5-HTP/5-HT et (2) à suggérer l'existence de variations circadiennes de ce « pool » indolique. Les recherches biochimiques ultérieures permetttront de préciser l'amplitude de ces variations nycthémérales de même que celle des composés de l'anabolisme et du catabolisme de la 5-HT. 
La réaction argentaffine qui avait permis, à l'appui d'autres techniques, de détecter la richesse en composés réducteurs (plus particulièrement la 5-HT) dans le compartiment granulaire (vésicules à cœur dense) des photorécepteurs rudimentaires de Sauropsidés (Juillard et Collin, 1976 ; Collin ef al., 1977) n'a pas conduit à révéler, chez le Brochet, les précipités susceptibles d'identifier cette indolamine. Des résultats négatifs avaient également été enregistrés dans les pinéalocytes de Rat par la méthode chromaffine (Jaim-Etcheverry et Zieher, 1968) alors que cetfe espèce, comme le Brochet, montre un taux élevé de 5 -HT et des vésicules à cœur dense très rares. De façon vraisemblable, la différence avec les Sauropsidés s'explique par une insuffisance de sensibilité des méthodes argentaffine et chromaffine qui ne révèlent la 5-HT que dans des fractions granulaires, c'est-à-dire là où son « uptake » et son stockage peuvent être très importants. II faut cependant rappeler que Jaim-Ełcheverry et Zieher (1968) étaient parvenus à identifier la fraction vésiculaire de 5-HT dans les terminaisons sympathiques du Rat.

L'ensemble de nos résultats, contrairement à ceux acquis chez certains Sauropsidés, et en accord avec ceux obtenus chez la Grenouille (Oksche et Hartwig, 1974 ef réf. correspondantes), est en faveur de l'existence d'un « pool » agranulaire d'indolamines du type 5-HTP/5-HT.

2) L'activité monoamine oxydasique (MAO). - Considérant, dans l'organe pinéal des Vertébrés, d'une part la richesse en monamines et, d'autre part, leur importance fonctionnelle, il étaił intéressant d'examiner la localisation cellulaire de l'activité MAO. Rappelons que l'existence de deux types de MAO ( $\mathrm{A}$ et $\mathrm{B}$ ) a été proposée (réf. in Juillard et Collin, 1979) mais comme ni le substrat, ni l'inhibiteur utilisés, ne sont spécifiques, ils ne permettent de les distinguer (discussion in Juillard et Collin, 1979). Ainsi nous parierons foujours de «l'activité MAO », dont Axelrod (1966) a souligné l'importance dans le catabolisme intracellulaire des monoamines du système nerveux central.

L'activité MAO, ayant parallèlement fait l'objet d'études chez le Brochet, le Lézard des murailles et la Perruche, nous allégerons volontairement la discussion, celle de Juillard et Collin (1979) devant être considérée comme complémentaire.

a) Sites de la désamination oxydative. En microscopie photonique, l'activité enzymatique paraît, chez le Brochet, être localisée au moins dans deux types cellulaires (CTP et CIE) de l'épithélium épiphysaire. Nos observations, effectuées à $10 \mathrm{~h}$ et à $16 \mathrm{~h}$, sur un nombre restreint d'individus ne permettent pas de garantir définitivement que les variations importantes enregistrées à ces deux périodes du nycthémère se rapportent soit à des variations individuelles importantes, soit à un rythme circadien de l'activité MAO. Ce point sera intéressant à préciser ultérieurement d'autant que l'importante désamination oxydative à $16 \mathrm{~h}$. s'accorde pleinement avec les variations nycthémérales des fluorophores jaunes correspondant au 5-HTP/5-HT dans l'épiphyse de Brochet ef avec les données chez le Pigeon et le Rat (revue de Quay, 1974) où les variations circadiennes du taux de l'acide 5-hydroxy-indole-acétique (5-HIAA, voir ci-dessous) suivent de près celles de la 5 -HT. Il faut faire remarquer cependant que chez le Rat, la MAO, à l'inverse d'autres enzymes pinéaliennes, ne semble pas avoir un rythme circadien d'activité.

Dans la région moyenne qui a été étudiée en microscopie électronique, l'activité 
MAO siège dans les deux types cellulaires : CTP ef CIE. La localisation intracellulaire de l'activité enzymatique est identique chez le Brochet, le Lézard et la Perruche (Juillard et Collin, 1979) : membrane mitochondriale externe, membranes interne et externe de l'enveloppe nucléaire, membranes du réticulum endoplasmique. En outre, les cellules de l'organe pinéal du Brochet (surtout les CTP), présentent une activité MAO au niveau des membranes plasmiques. L'ensemble de nos données ultracytochimiques est donc en parfait accord avec les conclusions des biochimistes qui, dans d'autres organes, ont proposé la localisation de l'activité MAO sur les membranes des constituants cellulaires cités ci-dessus (réf. in Müller et Da Lage, 1977). La validité de notre protocole expérimental et les réactions apparemment incomplètes sur les membranes sont discutées dans un autre travail (Juillard et Collin, 1979).

b) Substrats ef rôle de la MAO. Dans l’organe pinéal des Amniotes, la 5-HT, élaborée par les cellules intrinsèques, ef la noradrénaline des terminaisons des fibres orthosympathiques constituent les (ou des) substrats de la MAO (Juillard et Collin, 1979). Dans le cas du Brochet (et des autres Téléostéens), la nature orthosympathique de rares fibres catécholaminergiques localisées dans le tissu conjonctif méningé n'est pas établie. Il en est de même pour leur neurotransmetteur ; l'existence d'adrénaline (Owman et Rüdeberg, 1970) nécessite d'être confirmée.

Ainsi, il apparaît que pour la MAO membranaire des CTP et CIE, la 5-HT représente ici (voir aussi discussion antérieure) le principal substrat endogène ; il existe une relation étroite de localisation cellulaire entre l'enzyme et son substrat. Chez les Vertébrés supérieurs (Wurtman et al., 1968 ; Quay, 1974), il est bien connu que la voie métabolique principale de la 5-HT est celle de la désamination oxydative aboutissant à la formation de 5-HIAA. De plus, chez les homéothermes, une autre voie utilisant la désamination oxydative conduit à la formation du 5-hydroxytryptophol qui, après méthylation, est à l'origine du 5-méthoxyłryptophol. Ce dernier composé, physiologiquement moins actif que la mélatonine, aurait des propriétés voisines, au moins chez les Mammifères (Minneman ef Wurtman, 1975). Si la production de 5-HIAA par les CTP et les CIE est ici très hautement probable, celle du 5-hydroxytryptophol demeure, de même que ses sites de formation, encore hypothétiques chez les Vertébrés inférieurs.

L'ensemble de ces résultats souligne déjà l'intérêł que présentera ultérieurement l'étude biochimique de l'activité MAO (rythme circadien éventuel d'activité, à comparer à celui de son substrat, la 5-HT) et des composés dérivés de la désamination oxydative dans un organe à potentialité photoréceptrice certaine.

3) Conclusion. - Des résultats cytochimiques (méthode de Falck) ef pharmacologiques obtenus en automne et en hiver, il ressort que le «pool » agranulaire de 5-HTP/5-HT (précurseur de la mélatonine) produit par l'organe pinéal du Brochef est stocké (1) pringcipalement dans les différents types de CTP (cellules appartenant à la lignée sensorielle = réceptrice : Collin, 1971, 1979) et (2) également dans les CIE. La réaction argentaffine, contrairement à celle observée chez cerfains Sauropsidés ne nous a pas permis de révéler la localisation subcellulaire de la 5-HT. La présence du précurseur de la mélatonine dans les photorécepteurs typiques paraît compatible avec l'existence de cellules photoneuroendocriniennes (voir introduction ; concept à développer ultérieurement). Les variations régionales concernant le stockage de 5-HTP/5-HT suggèrent certaines différences dans l'activité métabolique indolique 
des CTP et des CIE, apparemment indépendantes de l'état de différenciation des CTP (Falcon, 1979b). L'étude cytochimique suggère également l'existence d'un ryth me nycthéméral du contenu en 5-HTP/5-HT avec un maximum pendant la période diurne (ici jours courts).

De rares fibres catécholaminergiques, observées dans les structures conjonctives méningées pourraient correspondre aux terminaisons à vésicules à contenu clair et dense détectées en microscopie électronique (Falcon, 1979a). II reste à établir si ce type d'innervation correspond à celle, orthosympathique, qui se développe pleinement à partir des Reptiles (Collin, 1969).

La monoamine oxydase (MAO), utilisant de toute vraisemblance essentiellement la 5-HT endogène comme substrat, a ses sites d'activité localisés dans les CTP et les CIE. A l'échelle ultrastructurale ces sites sont tout à fait semblables à ceux décrits parallèlement chez deux Sauropsidés (Juillard et Collin, 1979). Mais, chez le Brochet, une activité MAO existe en plus au niveau de membranes plasmiques.

L'ensemble de ces données s'inscrit dans le cadre des recherches que nous poursuivons sur les sites cellulaires du métabolisme indolique chez le Brochet.

Reçu en juin 1979,

Accepté en juilleł 1979.

Remerciements. - Ce travail a pu être réalisé grâce à une allocation d'étude DGRST (attribuée à J. Falcon), à un contrat DGRST no 76.7 .0043 et l'aide financière du CNRS. Nous remercions également Mlle D. Decourt qui a assuré la dactylographie de ce mémoire.

\section{References}

AXELROD J., 1966. Methylation reactions in the formation and metabolism of catecholamines and other biogenic amines. Phormacol. Rev., 18, 95-113.

BJÖRKLUND A., FALCK B., LINDVALL O., 1975. Microspectrofluorometric analysis of cellular monoamines after formaldehyde or glyoxylic acid condensation, 249-294. In BRADLEY P. B., Methods in brain research, John Wiley \& sons, London.

COLLIN J. P., 1969. Contribution à l'étude de l'organe pinéal. De l'épiphyse sensorielle à la glande pinéale : modalités de transformation et implications fonctionnelles. Ann. Stat. Biol. Besse-enChandesse, suppl. 1, 1-359.

COLLIN J. P., 1971. Differentiation and regression of the cells of the sensory line in the epiphysis cerebri, 79-120. In WOLSTENHOLME G. E. W., KNIGHT J., The pineal gland, Churchill Livingstone, Edinburg-London.

COLLIN J. P., 1979. Recent advances in pineal cytochemistry. Evidence of the production of indoleamines and proteinaceous substances by rudimentary photoreceptor cells and pinealocyfes of Amniota. Prog. Brain Res., 52, (sous presse).

COLLIN J. P., JUILLARD M. T., FALCON J., 1977. Localization of 5-hydroxytryptamine and protein(s) in the secretion granules of the rudimentary photoreceptor cells in the pineal of Lacerta. J. Neurocytology, 6, 541-554.

FALCK B., HILLARP N. A., THIEME G., TORP A., 1962. Fluorescence of catecholamines and related compounds condensed with formaldehyde. J. Histochem. Cytochem., 10, 348-354.

FALCON J., 1979a. L'organe pinéal du brochet (Esox lucius, L.). I. Etude anatomique et cytologique. Ann. Biol. anim. Bioch. Biophys., 19, 445-465.

FALCON J., 1979b. L'organe pinéal du brochet (Esox lucius, L.). II. Etude en microscopie électronique de la différenciation et de la rudimentation partielle des photorécepteurs ; conséquences possibles sur l'élaboration des messages pholosensoriels. Ann. Biol. anim. Bioch. Biophys., 19, 661-688. 
FALCON J., 1979c. Unusual distribution of neurons in the pike pineal organ. Prog. Brain Res., 52, (sous presse).

FALCON J., MOCQUARD J. P., 1979. L'organe pinéal du Brochet (Esox lucius, L.). III. Voies intrapinéales de conduction des messages photosensoriels. Ann. Biol. anim. Bioch. Biophys., 19, 1043-1061.

GABE M., 1968. Techniques histologiques. Masson et Cie, Paris, 649-651.

HAKANSON R., OWMAN CH., SPORRONG B., SUNDLER F., 1971. Electron microscopic classification of amine-producing endocrine cells by selective staining of ultra-thin sections. Histochemie, 27, 226-242.

JUILLARD M. T., COLLIN J. P., 1976. L'organe pinéal aviaire : étude ultracytochimique et pharmacologique d'un «pool » granulaire de 5-hydroxytryptamine chez la Perruche (Melopsittacus undulatus, Shaw). J. Microsc. Biol. cell., 26, 133-138.

JUILLARD M. T., COLLIN J. P., 1979. Membranous sites of oxidative deamination : a comparison between ultracytochemical and radioautographic studies in the pineal organ of the wall lizard and the parakeet. Biol. Cell., 36, 29-35.

JAIM-ETCHEVERRY G., ZIEHER L. M., 1968. Cytochemistry of 5-hydroxytryptamine at the electron microscope level. Il. Localization in the autonomic nerves of the rat pineal gland. $Z$. Zellforsch., 86, 393-400.

MEINIEL A., 1979. Detection and localization of biogenic amines in the pineal complex of Lampetra planeri (Petromyzontidae). Prog. Brain Res., 52 (sous presse).

MINNEMAN K. P., WURTMAN R. J., 1975. Effects of pineal compounds on mammals. Life Sci., 17, $1189-1200$.

MÜLLER J., DA LAGE C., 1977. Ultracytochemical demonstration of monoamine oxidase activity in nervous and non-nervous tissues of the rat. J. Histochem. Cytochem., 25, 337-348.

OKSCHE A., 1971. Sensory and glandular elements of the pineal organ, 127-146. In WOLSTENHOLME G. E. W., KNIGHT J., The pineal gland, Churchill Livingstone, Edinburg-London.

OKSCHE A., HARTWIG H.-G., 1974. Photoneuroendocrine systems and the third ventricle, 40-53, In KNIGGE K. M., SCOTT D. E., KOBAYASHI H., ISHII S., Brain Endocrine Inferaction II. S. Karger A. G., Basel.

OKSCHE A., HARTWIG H.-G., 1979. Pineal sense organs : components of photoneuroendocrine systems : Prog. Brain Res., 52 (sous presse).

OWMAN C., RÜDEBERG C., 1970. Light, fluorescence and electron microscopic studies on the pineal organ of the pike Esox lucius, with special regard to 5-hydroxytryptamine. Z. Zellforsch., 107, 522-550.

PETIT A., 1976. Contribution à l'éfude de l'épiphyse des reptiles : le complexe épiphysaire des Lacertiliens ef l'épiphyse des Ophidiens. Efude embryologique, structurale, ultrastructurale; analyse qualitative et quantitative de la sérotonine dans des conditions narmales et expérimentales. Th., Univ. Lovis Pasteur, Strasbourg, 205 pp.

QUAY W. B., 1966. Rhythmic and lighti-induced changes in levels of pineal 5-hydroxyindoles in the pigeon (Columba livia). Gen. comp. Endocr., 6, 371-377.

QUAY W. B., 1974. Pineal chemistry. Charles C. Thomas, Springfield, 430 pp.

SHANNON W. A. Jr., WASSERKRUG H. L., SELIGMAN A. M., 1974. The ultrastructural localization of monoamine oxidase (MAO) with tryptamine and a new tetrazolium salt, 2-(2'-benzothiazolyl)-5-styryl-3-(4'-phtalhydrazidyl) tetrazolium chloride (BSPT). J. Histochem. Cytochem., 22, 170-182.

VIVIEN-ROELS B., 1976. L'épiphyse des Chéloniens. Etude embryologique, siructurale ef ultrastrucfurale ; analyse qualitative et quantitative de la sérotonine dans les conditions normales ef expérimentales. Th. Univ. Louis Pasteur, Strasbourg, 189 Pp.

VIVIEN-ROELS B., PETIT A., ARENDT J., BRADTKE J., 1978. Circannual variations of the level of pineal indoleamines (serotonin, melatonin) and of their circadian fluctuations in reptiles. J. Neural Transm., Suppl. 13, 403.

WILHOFT D. C., QUAY W. B., 1965. Effects of temperature on brain contents of 5-hydroxytryptamine and related indoles in a lizard, Sceloporus occidentalis. Comp. Biochem. Physiol., 15, 325338.

WURTMAN R. J., AXELROD J., KELLY D. E., 1968. The pineal. Acad. Press, New York, 199 pp. 